\title{
11 Neurowissenschaften und eine weitere linguistisch sensible Perspektive
}

\begin{abstract}
We have the same nervous system basically that chimps do. There is only one difference, which is: We've got like three times as many neurons, and what the genetic differences are, are genes having to do with the number of rounds of cell division during fetal brain development. Essentially, what that says is: Take a chimp brain fetally and let it go two or three more rounds of division and you get a human brain instead. And, out come symphonies, and ideologies, and hopscotch[.]
\end{abstract}

Sapolsky (2011)

\subsection{Klärendes zur neuroanatomischen Konstellation des Menschen}

Das vorliegende Kapitel führt im Rahmen der nachfolgenden Erläuterungen zwei rote Fäden voriger Abschnitte fort. Einerseits sind neurowissenschaftliche Perspektiven, welche sich innerhalb der Sprachursprungsforschung in argumentativer und spekulativer Hinsicht von großer Beliebtheit zeigen, knapp vorzustellen und als weitere Exempel einschlägiger methodischer Problematiken zu kommentieren, und zusätzlich soll andererseits nochmals die EmergenzHypothese aufgegriffen und dabei in den Kontext des modernen neurowissenschaftlichen Kenntnisstands gesetzt werden. Zuletzt ist eine Brücke zum ontogenetischen Spracherwerb zu schlagen, denn dort eröffnet sich eine weitere Perspektive mit potenziell wertvollen Einblicken auf den phylogenetischen Spracherwerb.

In Reaktion auf das obige Zitat Sapolskys könnte versucht werden, zu argumentieren, dass dessen Aussagen möglicherweise etwas übermäßig grobkörnig formuliert wurden und dass entgegen dieser Beschreibung durchaus Unterschiede zwischen dem Gehirn eines Schimpansen und dem eines Menschen jenseits einer simplen Vergrößerung existieren. Prinzipiell ist diesem Einwand zuzustimmen, denn Plastizität, Lateralisation und Dichte der neuronalen Vernetzung erfuhren im Verlauf spezifisch menschlicher Evolution durchaus eine Steigerung gegenüber dem Schimpansen, jedoch finden sich - verglichen mit sonstigen Menschenaffen bzw. auch allgemeiner allen weiteren Primaten - eine nennenswert erhöhte Plastizität, Lateralisation und evolutionäre Flexibilität bereits im Schimpansen (Gómez-Robles, Hopkins \& Sherwood 2013 sowie Gómez-Robles et al. 2016) und in der Tat scheint des Gehirn des Menschen vielmehr eine primär quantitative Skalierung des Schimpansen-Gehirns darzustel- 
len denn ein qualitativ umstrukturiertes Organ (vgl. auch Miller, Barton \& Nunn 2019).

Unter Berücksichtigung der Aussage Sapolskys sowie jener Literatur ergibt sich somit eine mögliche Interpretation, laut der sich aus der quantitativen Steigerung des Gehirnvolumens und damit der Neuronenzahl jene „evolutionären“ Veränderungen des normativen adulten menschlichen Gehirns ableiten lassen. Ein ontogenetisches neuronales Programm, welches Vernetzung sowie Lateralisation beinhaltet, findet sich nicht nur im Menschen. Die zu stellende Frage besteht damit darin, was geschieht, wenn dieses ontogenetische neuronale Programm unter dem Vorhandensein einer gesteigerten Neuronenzahl ablaufen kann. Große Plausibilität besitzt die Feststellung einer inhärent gesteigerten Vernetzung - womöglich einschließlich einer Erhöhung der Lateralisation. Eine Steigerung der neuronalen Vernetzung sowie des lateralisierten Aufbaus des Gehirns könnte in diesem Sinne eine direkte Folge der quantitativen Steigerung der Neuronenzahl darstellen, anstatt explizit genetisch fundiert sein zu müssen. Freilich ist damit nicht zu unterstellen, dass keinerlei sonstige Anpassungen geschehen seien, jedoch besteht hierin ein starkes Argument gegen die axiomatische Feststellung einer umfangreichen genetischen und in Folge neuronalen evolutionären Umgestaltung des Gehirns. Unterschiede zwischen adulten menschlichen Gehirnen zu denjenigen von Schimpansen könnten damit weitläufig ontogenetisch erklärt werden - woraus sich, wie nachfolgend zu erläutern ist, eine vorige Ausführungen unterstützende Perspektive ergibt.

Im Kontext dieser Feststellungen ist zu Sapolsky (2011) zurückzukehren und aufzuzeigen, dass dessen obiges Zitat abgekürzt wurde, um die daran anknüpfende Aussage hier mit zusätzlichem Gewicht vorbringen zu können, denn er fährt fort „[that w] hat that tells you is: With enough quantity, you invent quality.“ In anderen Worten kann auf Basis des Vorgebrachten attestiert werden, dass sich das Phänomen der Emergenz aus Sicht der Neurowissenschaften und mit Hinblick auf die Evolution höherer kognitiver Befähigungen im Menschen plausibel festhalten lassen kann. In diesem Zuge sind freilich die Ausführungen des vorhergehenden Kapitels als zusätzlich untermauert zu sehen, denn das dort skizzierte Szenario erfährt sowohl hinsichtlich der Emergenz-Hypothese als auch hinsichtlich der Hypothese einer metakognitiven Steigerung im Kontext jener neuroanatomisch orientierten Feststellungen eine zusätzliche Fundierung.

Dies steht bereits prinzipiell im Kontrast zur unbedingten bzw. primären sprachlichen Bedeutung anderer vorgeschlagener neuroanatomischer Phänomene wie den Spiegelneuronen und der Lateralisation des menschlichen Gehirns. Zwar müssen diese durchaus eine Rolle auf dem Weg zum modernen 
Menschen gespielt haben und sind vermutlich sowohl in der phylogenetischen als auch in der ontogenetischen Ausbildung von Sprache involviert, denn anders lässt sich deren evolutionär persistente strukturelle und funktionale Rolle sowohl im Menschen als auch bei nicht-menschlichen Tieren nicht erklären. Jenseits dieser Feststellungen stellen diese Forschungsbereiche innerhalb der Diskussionen in der einschlägigen Literatur jedoch im Sinne bereits vorgestellter problematischer methodischer Vorgehensweisen Exempel einer pathologischen Argumentation dar. Aus diesem Grund soll nachfolgend im Kontext des gegebenen Methodenkatalogs eine knappe Aufbereitung geschehen.

\subsection{Zur Entdeckung der Spiegelneuronen und der Rolle der Lateralisation des Gehirns}

Spiegelneuronen gerieten in den 1990er Jahren in den Fokus mancher Sprachursprungstheoretiker und fanden in ähnlicher Weise wie das FOXP2-Gen zügig einseitige Auslegungen nicht nur bei fachfremden Autoren, sondern auch durch manche der ursprünglichen Entdecker (vgl. Rizzolatti \& Arbib 1998, Rizzolatti \& Craighero 2004 sowie Arbib 2000, 2005, 2008 und 2012). Einschlägige Studien stellten dabei fest, dass im Affen - und in Extension vermutlich im Menschen, wie auch in späteren Studien inferiert bzw. nachgewiesen wurde (bspw. Buccino, Binkofski \& Riggio 2004 sowie Mukamel et al. 2010) - Neuronen existieren, deren primäre Funktion darin besteht, beim Beobachten einer Handlung in gleicher, wenn auch abgeschwächter, Weise zu aktivieren wie beim Durchführen einer Handlung. Die damit verbundene Interpretation der Autoren besteht darin, dass das Aktivitätsmuster dieser Neuronen eine mentale Repräsentation der Handlung unabhängig davon hervorruft, ob sie selbst durchgeführt oder bei anderen beobachtet wird. Die darauf folgende Spekulation zu den potenziellen Funktionen reicht von basalen und höchst plausiblen Feststellungen darüber, dass das Spiegelneuronensystem helfe, Verhaltensweisen sowie -intentionen zu verstehen und Imitation zu unterstützen, bis zu sehr konkreten und mit größerer Entfernung abgeleiteten Aussagen dazu, dass damit ein Hinweis zugunsten einer ursprünglich gestischen Kommunikation im Frühmenschen gegeben sei (vgl. nochmals die soeben Erwähnten, aber auch andere wie Armstrong \& Wilcox 2007, insb. die Seiten 15, 28-29 und 54, Armstrong 2008, insb. Seite 302, sowie Corballis 2002, insb. die Seiten ab 46 und 214).

Dabei wird das einschlägige Szenario im Groben derart aufgespannt, dass auf Basis dieses Spiegelneuronensystems Gesten als sichtbare Form der Kommunikation von ebendiesem System hätten profitieren können, während orale Kommunikation nicht oder in geringerem Maße gefördert worden wären. Wie 
Rizzolatti \& Arbib (1998: 188) schreiben: „[S]uch an observation/execution matching system provides a necessary bridge from ,doing to ,communicating'[.]“ Ein besonderes Augenmerk liegt dabei auf dem Umstand, dass jene Spiegelneuronen im Rahmen früher Nachweise in nicht-menschlichen Affen in einem Bereich nachgewiesen wurden, welcher als homolog zum Broca-Areal des Menschen gesehen werden kann (vgl. Rizzolatti \& Arbib 1998: 189-190). Innerhalb der Literatur zum gestischen Sprachursprung verbreitete sich in Folge die Auffassung, dass hier hohe Bedeutsamkeit festzustellen sei, weitläufig. Aus Corballis (2002: 46): „This reinforces speculation that mirror neurons constitute a precursor to language, which also requires a mapping between the production and the perception of complex actions." Diesem Fokus hinsichtlich vermeintlicher Rollen der Spiegelneuronen sowie der Verortung im Gehirn folgten entsprechende Schlüsse (wiederum Corballis 2002: 46): „It will not have escaped the reader's attention, as it did not escape Rizzolatti's, that the actions are manual, not vocal, strongly suggesting a gestural origin for language.“

Die Literatur der relevanten Einzelwissenschaften zeigt sich jedoch sowohl als kritisch gegenüber der Implikation von komplexen kognitiven Phänomenen als direkte Folge von Spiegelneuronen als auch als weitläufig durchwachsen hinsichtlich vieler Einzelheiten, welche für die vorgestellten Argumente von nennenswerter Wichtigkeit sind. So wurden der Verbindung zwischen Spiegelneuronen und „action understanding“ sowie daran angeschlossener, hierarchisch hoher Phänomene nicht nur bestätigende Meinungen entgegengebracht, sondern auch umfassend Skepsis (vgl. bspw. Dinstein et al. 2008, Hickock 2009, Pascolo, Budai \& Rossi 2010 und Koksogonov 2012). Keysers \& Gazzola (2010: R353) weisen darauf hin, dass anscheinend keine „Zentren“ für Spiegelneuronen existieren, sondern dass sie mit unerwartet geringer Intensität großflächig vorkommen: „These results suggest we should stop considering certain brain regions as intrinsically ,mirror neuron regions'[.]“ Daraus folgt freilich eine wenigstens teilweise Relativierung der angeblichen besonderen Bedeutung von Spiegelneuronen in zum Broca-Areal homologen Zentren bei nichtmenschlichen Affen.

Weiterhin sind Spiegelneuronen als ein Phänomen wohl im Tierreich weit verbreitet, was die vorgeschlagene enge Verbindung mit Sprachevolution weiter schwächt, und sind daneben anscheinend Teil verschiedener neuronaler Systeme, welche nicht nur motorischer, sondern auch sozial-interpretativer Natur sind, sodass eine eng gesteckte Funktion zunehmend unwahrscheinlich erscheint (Ferrari \& Rizzolatti 2014, Ferrari et al. 2017, Cacioppo, Bolmont \& Monteleone 2018). Zuletzt involvieren Prather, Okanoya \& Bolhuis (2017) und weiterführend Giret, Edeline \& Del Negro (2017) Spiegelneuronen in Vögeln mit deren 
Vokalisationen bzw. der Erlernung ebendieser Vokalisationen und zeigen damit, dass entgegen dem zuvor beschriebenen Argument auch im Menschen nicht zwingend eine gestische Phase existiert haben muss, um die Existenz bzw. Relevanz des Spiegelneuronensystems im Menschen zu erklären.

Mithin offenbart sich ein Gesamtbild, in welchem Spiegelneuronen ein durchaus bedeutsames Phänomen darstellen, denn anders lässt sich wie zuvor angemerkt deren umfangreiche Existenz in verschiedensten Taxa und die umfassende Integration in einer Vielzahl neuronaler struktureller sowie funktionaler Komplexe kaum erklären. Dennoch lassen sich Spiegelneuronen zugleich nicht als primäre evolutionäre Faktoren in der Ausbildung von Sprache - geschweige denn innerhalb eines gestischen Sprachursprungs - klassifizieren. Tatsächlich zeigt sich ein ähnliches Bild wie bei vorhergehenden, die Genetik betreffenden Erläuterungen: Spiegelneuronen sind funktional weitläufig involviert, sind jedoch potenziell in keiner einzigen Domäne der primäre funktionale Faktor, sondern liegen eingebettet in einem komplexen funktionalen Geflecht, in welchem sie beitragend teilhaben. Das aktuelle Forschungsfeld ergibt eine Reihe interessanter Perspektiven und fruchtbarer Ansätze für eine weiterführende Forschung, jedoch ist der Forschungsstand nicht bzw. noch nicht an einem Punkt angelangt, an dem konkrete Interpretationen mit ausreichender Sicherheit festgehalten werden können.

Gleiches gilt prinzipiell für die Lateralisation des Gehirns, welche sich bei einer normativen adulten Sprachbefähigung insbesondere auch hinsichtlich sprachlicher funktionaler Zentren zeigt, womit die Unterstellung einer hohen Wichtigkeit für die Ausbildung derselben nahe liegt (für ein jüngeres einschlägiges Review vgl. Ries, Dronkers \& Knight 2016). Auch wenn dieses Phänomen innerhalb der jüngeren Sprachursprungsforschung nicht die gleiche argumentative Popularität innehält wie die Entdeckung und Erforschung von Spiegelneuronen, so eröffnet sich dennoch eine ausgiebige Diskussion (vgl. bspw. Corballis 2002: 165-183), welche jedoch wiederum in ähnlicher Weise charakterisiert werden kann wie zuvor vorgestellte Forschungsbereiche. In anderen Worten existieren sowohl eine besondere Ausprägung im Menschen als auch eine wichtige strukturelle und funktionale Implikation in kognitive sowie konkret sprachliche neuronale Komplexe bei der gleichzeitigen Feststellung, dass hier kein ausreichend starkes Argument dafür besteht, dass hierin die ausschlaggebende Besonderheit besteht, welche zur modernen menschlichen Sprachfähigkeit hätte führen können. Dies darum, da es sich wiederum um ein im Tierreich weitläufig existierendes Phänomen mit weitläufiger Funktionalität jenseits der sprachlichen Dimension handelt (vgl. als Review mit teilweise direkter Antwort auf Corballis 2002 Halpern et al. 2005). Im Sinne der soeben 
gemachten Feststellungen und mit jenem Verweis auf zweifelnde Perspektiven sei an gegebener Stelle eine erweiterte Diskussion zu vermeiden. Die generelle Mahnung einer übermäßigen Generalisierung bzw. Idealisierung im Sinne des Fokus-Effekts sei jedoch festgehalten.

\subsection{Selektive Einflüsse auf die Evolution der menschlichen Neuroanatomie}

Damit ist nochmals auf die um Sapolsky (2011) explizierte Situation zurückzukehren. Jener erläutert, dass die geringen genetischen Unterschiede, die zwischen dem Schimpansen und dem Menschen existieren, primär mit dem Geruchssinn, dem Haarwuchs, dem Knochenbau und der Muskulatur zu tun haben und dass die wenigen auf die neuronale Entwicklung gerichteten Unterschiede primär quantitativer Natur sind. Daraufhin stellt sich sicherlich die Frage, was diese Vergrößerung des Gehirnvolumens verursacht haben könnte, denn es bedarf einer adäquaten Begründung im Sinne eines Selektionsdrucks mit Hinblick auf diese evolutionäre Entwicklung. Ein dankbares Erklärungsmodell ergibt sich über die ökologischen Veränderungen während der Evolutionsgeschichte der Australopithecinen hin zur Gattung des Menschen. Ein klimatischer Langzeittrend zu zunehmender Trockenheit über mehrere geologisch gesehen kurzfristige Schwankungen zwischen relativer Feuchtigkeit und relativer Trockenheit von vor etwa 2,8 Millionen Jahren bis vor etwa 1,0 Millionen Jahren (vgl. deMenocal 1995 sowie Trauth et al. 2005) führte zu Veränderungen in Ernährungsstrategien für die damaligen Taxa. Zuvor war die Ernährung primär auf Früchte und ähnlich hochqualitative Pflanzennahrung fokussiert und glich damit vermutlich im Groben derjenigen des modernen Schimpansen, welche daneben auch gelegentlich Fleisch in ihre Ernährung integrieren (Aiello \& Wheeler 1995, Ferraro et al. 2013 sowie Martínez et al. 2016).

Im Verlauf der sukzessive größeren Trockenheit bzw. der dabei auftretenden klimatischen Fluktuationen entwickelten sich unter den Australopithecinen in Form robuster und graziler Formen zwei ökologische Nischen heraus. Mit Verweis auf das Kapitel der Menschwerdung, wo die zugrundeliegende Entwicklung etwas umfangreicher skizziert werden konnte, sei hier lediglich angemerkt, dass die Schwesterngattung des Menschen Paranthropus eine evolutionäre Entwicklung ihrer Ernährungsstrategie in Richtung qualitativ weniger wertiger, jedoch auch bei steigender Trockenheit umfassend verfügbarer, Pflanzenmaterialien durchmachte, während die Gattung des Menschen Anpassungen in Richtung eines zunehmenden und schließlich beständigen Fleischkonsums erfuhren (bspw. Stedman et al. 2004 sowie nochmals Ferraro et al. 2013). Als ein 
zunehmend karnivores Taxon, welches jedoch die Schnelligkeit, Kraft, Krallen und Befähigung zu Nachtsicht der in der Nahrungskette dominanten Großkatzen vermisste, waren jedoch besondere Verhaltensstrategien vonnöten, welche eine neuartige ökologische Nische eröffneten.

Als zentral können hier wenigstens drei Faktoren gesehen werden. Erstens bedingte die zuvor auf Früchten basierende Ernährung ein hochauflösendes und farbintensives Sehen. Diese hervorragenden Augen erleichtern in der sich zunehmend aufspannenden Savanne die Suche und potenziell die Jagd von Beute. Zweitens begründen die durch das Klettern flexiblen und starken Hände eine Befähigung, Werkzeuge zu produzieren und $\mathrm{zu}$ verwenden - eine Verhaltensweise, die sich wie zuvor erläutert bereits unter den grazilen Australopithecinen etablierte und in Homo extensiv betrieben wurde. Drittens eröffnete ein relativ voluminöses Gehirn das Potenzial all jener Verhaltensstrategien, welche sich im Menschen einstellten: Besondere Leistungsfähigkeit in mechanischer sowie sozialer Intelligenz, in der Planung, bei der Kooperation sowie Kommunikation und allem Weiteren. Aus diesen Faktoren ergaben sich Beschaffungsstrategien, in welchen Planung, Koordination und potenziell Oralität eine wichtige Rolle spielten. Ganz allgemein etablierte sich ebenjene Variabilität des Verhaltens, welche den Kern der menschlichen Erfolgsgeschichte stellt.

Es zeigt sich dabei wiederum ein kohärent stützendes Zusammenspiel mit dem in vorigen Kapiteln Erläuterten. Im soeben aufgezeigten Kontext greifen anthropologische Beobachtungen bzw. Szenarien wie in Knight \& Lewis (2017) besonders fruchtbar, denn die bereits vorgestellten anthropologischen Beobachtungen sowie die Möglichkeit von ,aggressive vocal mobbing“ (vgl. auch frühere Veröffentlichungen wie Eibl-Eibesfeldt 1989) gegenüber Lebewesen außerhalb der eigenen Gruppe eröffnen Szenarien der steigenden Oralität insbesondere unter Zuhilfenahme der Konzepte der Metakognition sowie der Emergenz, welche Mechanismen beschreiben, nach denen eine biologische Kontinuität mit einer kognitiven und sprachlichen Diskontinuität in einem übergreifenden Erklärungsansatz vereint werden können. Auch die in vorigen Kapiteln vorgestellten genetischen, demographischen und nicht zuletzt paläoanthropologischen sowie archäologischen Erkenntnisse stellen sich hinzu und zeichnen ein komplementäres Bild einer kulturellen Kontinuität in einer reichen demographischen Dynamik. 


\subsection{Die Rolle des ontogenetischen Spracherwerbs in der Sprachursprungsforschung}

Ein letztes - weil in letzter Konsequenz umfassend neuronal begründetes Phänomen bzw. eine letzte entsprechende Perspektive soll an gegebener Stelle noch skizziert und in die Diskussion eingebracht werden, ohne dass allzu umfangreich dort verblieben werden soll. Der ontogenetische Spracherwerb stellt zum Trotz seiner scheinbaren Banalität eine außerordentliche Leistung dar. Das Kind steht vor der monumentalen Aufgabe, ohne die vorhergehende Ausbildung höherer kognitiver Fähigkeiten sprachliche Kategorien sowie deren Merkmale zu erkennen, zu erlernen, in hierarchische Relationen zu setzen bzw. ineinander $\mathrm{zu}$ integrieren und letztendlich sprachliche Einheiten $\mathrm{zu}$ produzieren. Obwohl die dabei involvierten Mechanismen umfassend beforscht werden (vgl. das Review von Kuhl 2010), so ist eine vollständige Erschließung dieses Prozesses im aktuellen Kenntnisstand noch nicht möglich. Der nichtsdestotrotz erreichte, umfangreiche Wissensstand könnte trotz vorhandener Lücken von konkretem Interesse für den phylogenetischen Spracherwerb sein.

Wie Brentari \& Coppola (2012: 201) feststellen, besteht ein umfassender Fokus der einschlägigen Forschung auf der Erforschung nicht-menschlicher Tiere: „Because all preceding species of humans have disappeared, some researchers have looked to great apes, our genetic relatives, for an explanation of language evolution." Wie im Verlauf der vorliegenden Arbeit wiederkehrend betont wurde, ist jedoch der konkrete Vergleich nicht-menschlicher Kommunikationssysteme mit sprachlichen Strukturen bzw. der linguistischen Terminologie als problematisch $\mathrm{zu}$ sehen. Hier ist ein weiterer Gedanke Brentari \& Coppolas (wiederum 2012: 201) aufzugreifen: „Language acquisition provides another important window on this question; in a certain sense every child creates a language anew as she/he matures and acquires the language of those around her/him.“ Nun ist anzumerken, dass zwar Veröffentlichungen existieren, welche sich in diesem Sinne mit den protosprachlichen Entwicklungen im Erstspracherwerb mit Bezug auf die Evolution von Sprachbefähigung beschäftigen (vgl. bspw. Greenfield, Lyn \& Savage Rumbaugh 2008), jedoch besteht hier sicherlich eine im erweiterten Forschungskontext als nebensächlich $\mathrm{zu}$ bezeichnende Perspektive.

Diese Vernachlässigung gegenüber einer Integration des ontogenetischen Spracherwerbs als eine möglicherweise partiell treffende Vorzeichnung des phylogenetischen Spracherwerbs in die einschlägige Forschung soll hier als Versäumnis deklariert werden. Dieses begründet sich womöglich teilweise auf der umstrittenen Forschungsgeschichte zu Haeckels (1866) Rekapitulationsthe- 
orie bzw. seines biogenetischen Grundgesetzes, laut welchem die biologische Ontogenese eine verdichtete Rekapitulation der Phylogenese sei. In anderen Worten bestünde in der embryonalen Entwicklung eines Individuums ein stufenweises Durchlaufen der vorherigen stammesgeschichtlichen evolutionären Entwicklung. Während diese Aussagen intuitiv wenigstens im Groben Plausibilität zu haben scheinen, so führte Haeckels Auslegung als biologisches Gesetz sowie Versuche einer hintergründigen Erklärung dieses Gesetzes zu einem historisch großen Widerstand gegen diese Position. So schreibt Blechschmidt (1977: 32): „The so-called basic law of biogenetics is wrong. No buts or ifs can mitigate this fact. It is not even a tiny bit correct or correct in a different form, making it valid in a certain percentage. It is totally wrong." Manche Autoren verbrachten hunderte von Seiten damit, eine angemessen kritische Perspektive auf die Gültigkeit von Haeckels Thesen aufzuspannen (vgl. Gould 1977).

Parallel zur Diskussion einer biologischen Gültigkeit derartiger ontogenetischer Rekapitulationen der Phylogenese bestand jedoch auch eine vergleichbare Denktradition hinsichtlich der Kognition bzw. kulturellen Reife des Menschen als Art bzw. von Kindern als Individuen. So heißt es bei Spencer (1861: 76): „If there be an order in which the human race has mastered its various kinds of knowledge, there will arise in every child an aptitude to acquire these kinds of knowledge in the same order." Noch prägnanter fasst er den Gedanken wie folgt zusammen (Seite 5): „Education is a repetition of civilization in little.“ Dass dabei eine mit Haeckel höchst vergleichbare Formulierung gegeben ist, kann nicht bestritten werden. Auch im Verlauf des 20. Jahrhunderts bestand dieser Gedanke fort und bezog sich dabei mitunter auch konkret auf die Entwicklung der Sprachfähigkeit (bspw. Hall 1904 sowie Parker \& Gibson 1979), konnte sich jedoch nicht weitläufig durchsetzen. Es soll an gegebener Stelle postuliert werden, dass hier ein produktiver und nicht ausreichend genutzter Ansatz vorliegt - ohne dass an dieser Stelle ausreichend gezeigt werden könnte, ob diese Analogie einer umfassenden, kritischen Erschließung standzuhalten vermag. Die generelle Hypothese begründet sich jedoch auf mehreren, knapp zu skizzierenden Ebenen.

In einer ersten Feststellung zeigen Schimpansen parallel zu Kindern zu einem Alter von etwa zwei Jahren in wenigstens mehreren sprachlichen Dimensionen - prominent ist hier beispielsweise die Phonologie auszuklammern - und vor allem in kognitiver Hinsicht eine wenigstens vergleichbare Befähigung (vgl. bspw. Limber 1977, Greenfield \& Savage-Rumbaugh 1993 sowie Greenfield, Lyn \& Savage-Rumbaugh 2008). Als bemerkenswert zeigt sich im weiteren Verlauf insbesondere die Abruptheit der sich einstellenden Unvergleichbarkeit, sobald im kindlichen Spracherwerb gewisse Meilensteine erreicht werden, welche eine 
sofortige qualitative Unvergleichbarkeit gegenüber den Befähigungen von Schimpansen zur Folge haben. Zum Trotz dieser sich einstellenden Diskontinuität ist mit Interesse anzumerken, dass der kindliche Spracherwerb durchaus gewisse frühe Parallelen mit nicht-menschlichen Befähigungen aufweist.

Weiterhin schließt sich an die soeben geschehene Erwähnung von Meilensteinen im Spracherwerb eine potenziell gewichtige Beobachtung an: Im Verlauf des Erstspracherwerbs geschehen regelmäßig qualitative Innovationen sowohl in kognitiver als auch in sprachlicher Hinsicht, welche kein simples Kontinuum mit vorhergehenden kognitiven bzw. sprachlichen Stufen einzugehen scheinen. Stattdessen stellen sich anscheinend diskontinuierliche geistige Errungenschaften ein, welche konstitutionell neuartige und mitunter ökonomisch vorteilhafte Mechanismen respektive Strategien darstellen - und potenziell ist hier mindestens mit Hinblick auf die zugrundeliegende neuronale Landschaft eine Verbindung mit der Überschreitung von Schwellenwerten respektive einer Ausbildung neuartiger Qualitäten im Sinne der Emergenz herzustellen. Derartige sprachliche Fundamente wie die Ausbildung von Merkmalsystemen, welche Phänomenen wie dem kindlichen Vokabelspurt zu unterliegen scheinen, stellen die Grundlage für die sich im Erstspracherwerb hierarchisch aufbauenden sprachlichen Systeme (Jakobson \& Halle 1956, Chomsky \& Halle 1968).

Auf Basis der zuvor gemachten Feststellung, dass potenziell emergente Phänomene in kognitiver, neuronaler und in letzter Konsequenz sprachlicher Hinsicht im Verlauf der Menschwerdung eine Rolle gespielt haben könnten, ergibt sich im Kontext der sich darbietenden ontogenetischen Entwicklung potenziell ein mächtiges Werkzeug für einen Einblick in einen näherungsweise analogen Aufbau eines sprachlichen Systems. Es zeigt sich damit eine potenziell stützende Kompatibilität mit bereits erläuterten Konzepten sowie Szenarien und darüber hinaus eröffnet sich ein noch nicht ausreichend beachteter Forschungsbereich, welcher in zukünftigen Untersuchungen potenziell fruchtbar inkorporiert werden könnte. 